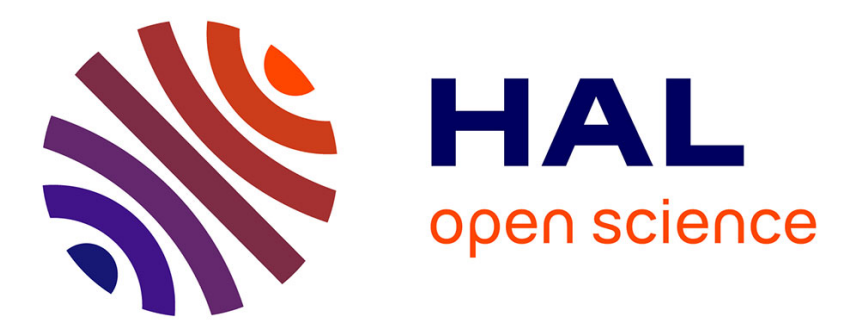

\title{
L'essai de compression plane de monocristaux encastrés : méthode d'étude du comportement d'un cristal soumis à une déformation plastique imposée
}

\author{
J.H. Driver, A. Skalli
}

\section{- To cite this version:}

J.H. Driver, A. Skalli. L'essai de compression plane de monocristaux encastrés: méthode d'étude du comportement d'un cristal soumis à une déformation plastique imposée. Revue de Physique Appliquée, 1982, 17 (8), pp.447-451. 10.1051/rphysap:01982001708044700 . jpa-00245021

HAL Id: jpa-00245021

https://hal.science/jpa-00245021

Submitted on 1 Jan 1982

HAL is a multi-disciplinary open access archive for the deposit and dissemination of scientific research documents, whether they are published or not. The documents may come from teaching and research institutions in France or abroad, or from public or private research centers.
L'archive ouverte pluridisciplinaire HAL, est destinée au dépôt et à la diffusion de documents scientifiques de niveau recherche, publiés ou non, émanant des établissements d'enseignement et de recherche français ou étrangers, des laboratoires publics ou privés. 


\title{
L'essai de compression plane de monocristaux encastrés : méthode d'étude du comportement d'un cristal soumis à une déformation plastique imposée
}

\author{
J. H. Driver et A. Skalli \\ Département de Matériaux et Ingénierie, \\ E.N.S.M.S.E., 158, cours Fauriel, 42023 Saint-Etienne Cedex, France
}

(Reçu le 3 mars 1982, révisé le 29 avril 1982, accepté le 7 mai 1982)

\begin{abstract}
Résumé. - Une méthode simple d'essai de compression plane de monocristaux encastrés est proposée pour l'étude de la plasticité cristalline dans des conditions de déformation plastique imposée. Le principe consiste à comprimer dans une matrice un cristal d'orientation $\mathbf{X}_{3}, \mathbf{X}_{2}$ (où $\mathbf{X}_{3}$ signifie le vecteur normal au plan de compression et $\mathbf{X}_{2}$ la direction d'allongement), encastré entre deux autres cristaux d'orientation $-\mathbf{X}_{3}, \mathbf{X}_{2}$. Des résultats expérimentaux sur des cristaux d'aluminium d'orientations différentes confirment la validité de la technique.
\end{abstract}

Abstract. - A simple technique for the plane strain compression of constrained surrounded crystals is proposed for crystal plasticity studies under conditions of imposed plastic deformation. The basic principle consists of deforming, by compression in a die, a crystal of orientation $\mathbf{X}_{3}, \mathbf{X}_{2}$ (where $\mathbf{X}_{3}$ indicates the vector normal to the compression face and $\mathbf{X}_{2}$ the direction of elongation), constrained by two other crystals of orientation $-\mathbf{X}_{3}, \mathbf{X}_{2}$. Experimental results obtained on aluminum crystals of different orientations confirm the validity of the technique.

1. Introduction. - Les études de la plasticité des cristaux sont effectuées, en général, par des essais classiques de traction, de compression, ou parfois, de cisaillement. Dans la plupart de ces expériences sur monocristaux d'orientation cristalline quelconque (les orientations de symétrie d'ordre 2, 3 ou 4 étant exclues), une composante seulement du tenseur de la déformation plastique, $\boldsymbol{\varepsilon}$, est imposée. Dans ce cas le critère de Von Mises [1], selon lequel $n$ systèmes indépendants de glissement sont nécessaires pour accommoder $n$ composantes imposées de $\varepsilon$, implique que seulement un système de glissement soit actif. Rappelons que le critère de Von Mises est couramment utilisé dans les théories générales de la plasticité cristalline, [2], [3], [4]. Or si l'on veut confirmer ces théories par l'expérience et, d'une façon plus générale, étudier le comportement d'un cristal d'orientation quelconque en glissement multiple, il convient de tester le cristal dans des conditions où plusieurs ou toutes les composantes du tenseur de déformation plastique sont imposées. Avec cet objectif, l'essai de compression plane a été appliqué ces dernières années à l'étude de la déformation plastique de monocristaux c.f.c. [5, 6] et c.c. [7].

Le principe de cet essai est présenté sur la figure 1. Le cristal est comprimé dans la direction $\mathbf{X}_{3}$ et s'allonge dans la direction $\mathbf{X}_{2}$. Dans le cas d'un monocristal

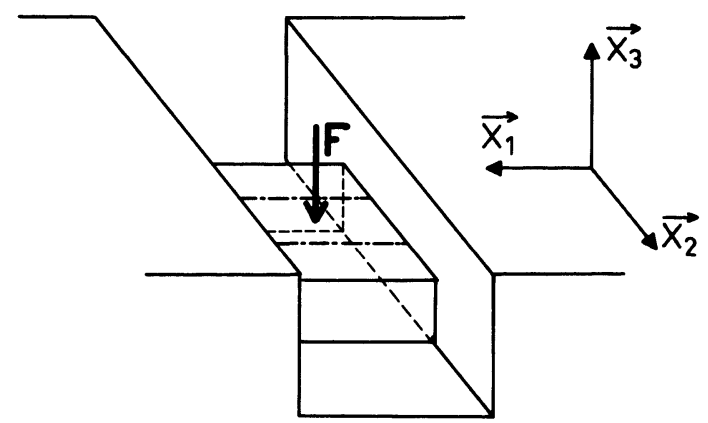

Fig. 1. - Schéma du dispositif de compression plane.

[Schematic diagram of the plane strain compression die.]

d'orientation quelconque les tenseurs des contraintes et des déformations ont alors la forme suivante :

\begin{tabular}{|c|c|c|c|}
\hline \multirow{3}{*}{ tenseur des contraintes $\sigma=$} & $\sigma_{11}$ & 0 & $\left.\sigma_{13}\right)$ \\
\hline & 0 & 0 & 0 \\
\hline & $\sigma_{13}$ & 0 & $\sigma_{33} /$ \\
\hline \multirow{3}{*}{$\begin{array}{l}\text { tenseur des déformations } \\
\text { plastiques } \varepsilon \text { (symétrique) }\end{array}$} & 0 & $\varepsilon_{2}$ & 0 \\
\hline & $\varepsilon_{21}$ & $\varepsilon_{22}$ & $\varepsilon_{23}$ \\
\hline & 0 & $\varepsilon_{23}$ & $\varepsilon_{33}$ \\
\hline
\end{tabular}


Trois termes indépendants de $\varepsilon$ sont imposés : $\varepsilon_{11}(=0), \varepsilon_{13}(=0)$ et $\varepsilon_{33}$. Par conservation de volume $\varepsilon_{11}+\varepsilon_{22}+\varepsilon_{33}=0$ et donc $\varepsilon_{22}=-\varepsilon_{33}$. Les deux cisaillements $\varepsilon_{21}$ et $\varepsilon_{23}$ ne sont pas imposés. Ces conditions mixtes résultent du fait que les composantes du tenseur des contraintes dans la direction $\mathbf{X}_{2}$ sont nulles (face libre), tandis que les trois composantes de déplacement de matière dans cette direction $\left(\varepsilon_{21}\right.$, $\left.\varepsilon_{22}, \varepsilon_{23}\right)$ sont possibles. Remarquons qu'il est possible de calculer le tenseur de contrainte $\sigma$ et, à l'aide de la loi de Schmid et Boas d'en déduire les systèmes de glissement actifs ainsi que les composantes $\varepsilon_{12}$ et $\varepsilon_{23}$ de la déformation, moyennant certaines hypothèses sur les amplitudes de glissement [4]. En général on trouve que 3 ou 4 systèmes de glissement sont susceptibles de glisser, ce qui permet l'étude du comportement plastique en glissement multiple sous conditions mixtes.

Or, il est parfois intéressant de pouvoir imposer 4 ou 5 termes indépendants de $\varepsilon$ en supprimant 1 ou 2 des cisaillements non imposés. L'objet de cette note est de montrer comment y parvenir par l'utilisation de monocristaux encastrés entre deux autres cristaux.

2. Méthode d'encastrement. - Le cristal à tester est rendu solidaire, aux deux extrémités correspondant à la direction $\mathbf{X}_{2}$ avec deux cristaux de même composition chimique, dont les cisaillements $\varepsilon_{21}^{\prime}$ et $\varepsilon_{23}^{\prime}$ seraient, si les cristaux n'étaient pas solidaires, d'amplitudes égales mais de signes opposés aux cisaillements du cristal central. Par commodité les deux cristaux d'encastrement sont de même orientation que le cristal central mais tournés de $\pi$ autour d'un axe $\mathbf{X}_{1}, \mathbf{X}_{2}$ ou $\mathbf{X}_{3}$ par rapport à ce dernier. Le principe de la technique s'explique par les propriétés de symétrie du cristal. La figure 2 montre, en deux dimensions qu'une rotation du cristal central B (initialement de même orientation que $A$ et $C$ ) de $\pi$ autour de l'axe $\mathbf{X}_{2}$ remplit la condition sur les cisaillements, de sorte que lorsque les cristaux sont solidaires la déformation de l'ensemble conduit à l'élimination mutuelle des cisaillements $\varepsilon_{21}$ et $\varepsilon_{21}^{\prime}$ par incompatibilité. En trois dimensions, la rotation de $\pi$ autour de l'axe $\mathbf{X}_{2}$ permet

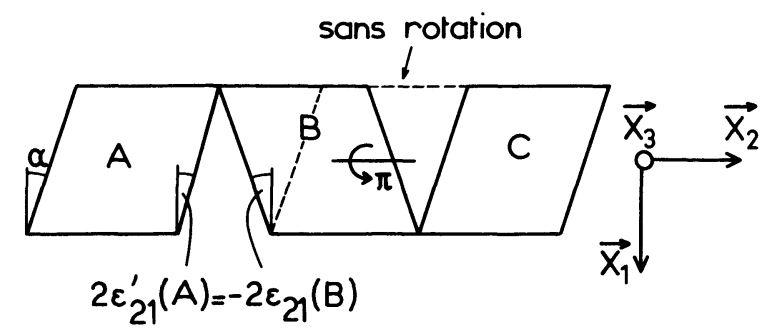

Fig. 2. - Schéma, en deux dimensions, de la relation d'orientation entre le cristal central (B) et les cristaux d'encastrement (A et $\mathrm{C}$ ) qui permet d'éliminer le cisaillement $\varepsilon_{21}$.

[Two dimensional schematic diagram of the orientation relationship between the centre crystal (B) and the outer crystals $(A$ and $C)$ which eliminates the shear strain $\varepsilon_{21}$.] également d'éliminer le cisaillement $\varepsilon_{23}$; les cinq termes indépendants du tenseur de déformation plastique du cristal central sont alors complètement imposés. De plus si l'on cherche à imposer quatre termes de $\varepsilon$ en n'éliminant que l'un des deux cisaillements $\varepsilon_{21}$ ou $\varepsilon_{23}$ il suffit de tourner le cristal central de $\pi$ autour d'un axe $\mathbf{X}_{1}$ ou $\mathbf{X}_{3}$ :

- une rotation de $\pi$ autour de $\mathbf{X}_{3}$ supprime $\varepsilon_{23}$ mais pas $\varepsilon_{21}$ et

- une rotation de $\pi$ autour de $\mathbf{X}_{1}$ supprime $\varepsilon_{21}$ mais pas $\varepsilon_{23}$.

D'une manière générale l'ensemble de trois cristaux orientés par ces opérations de symétrie sera appelé "tricristal symétrique incompatible".

En pratique nous avons élaboré en une seule opération des tricristaux d'aluminium $(99,996 \%)$ par solidification dirigée en utilisant des germes orientés tricristallins. Le germe tricristallin est obtenu, à partir d'un monocristal de l'orientation désirée, par découpage en trois parallélépipèdes le long de l'axe du cristal $\mathbf{X}_{1}$ (Fig. 3), suivi d'une rotation du parallélépipède central de $\pi$ autour de l'axe voulu (par exemple $\mathbf{X}_{2}$ ). Après élaboration par solidification dirigée, le tricristal est découpé en tranches (plan de découpage $X_{2} X_{3}$ voir figure 3) afin d'obtenir des éprouvettes tricristallines de compression plane.

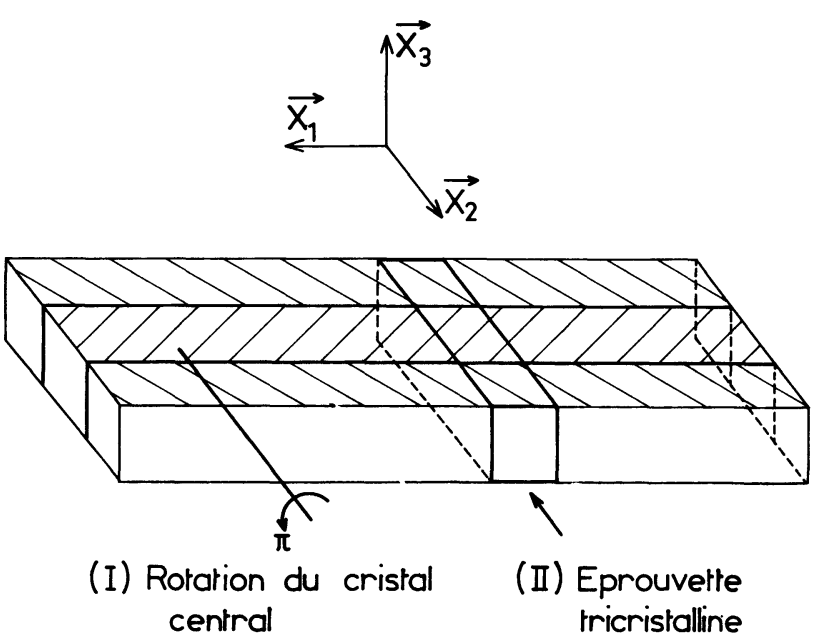

Fig. 3. - Technique de préparation d'une éprouvette tricristalline : (I) Découpage du monocristal et rotation du cristal central; (II) Elaboration du tricristal et découpage en éprouvettes de compression plane.

[Preparation technique of a tricrystal specimen : (I) Single crystal sliced into three and the centre crystal rotated; (II) Tricrystal sectionned into plane strain compression specimens.]

Notons qu'il est également possible d'élaborer des tricristaux orientés à partir d'un monocristal découpé en trois parties par soudage par bombardement d'électrons [8]. Nous avons réalisé quelques tricristaux d'aluminium par soudage mais leur qualité est infé- 
rieure à celles obtenues par solidification dirigée. Néanmoins la technique de soudage pourra s'appliquer à d'autres métaux et alliages.

3. Résultats expérimentaux. - Des essais de compression plane ont été réalisés sur monocristaux et cristaux encastrés pour vérifier la technique d'encastrement. Le dispositif de compression plane, qui comporte une matrice et un poinçon indéformables, est placé dans une machine classique de traction-compression. Les éprouvettes en aluminium de dimensions $7 \times 7 \times 15 \mathrm{~mm}$ subissent un traitement thermique (revenu $1 \mathrm{~h}$ à $450^{\circ} \mathrm{C}$ ) et un polissage électrolytique avant l'essai. Le frottement est fortement réduit par l'utilisation de films minces de téflon qui lubrifient toutes les surfaces de contact éprouvette-matrice. Au cours d'une expérience l'essai est interrompu périodiquement à des intervalles de déformation rationnelle d'environ 0,1 à 0,2 pour mesurer les dimensions de l'échantillon et renouveler le film de téflon [6]. Les cisaillements $\varepsilon_{21}$ et $\varepsilon_{23}$ sont mesurés par les angles $\alpha$ et $\beta$ que font des lignes de repères, initialement orthogonales, tracées sur l'échantillon dans les directions $\mathbf{X}_{1}, \mathbf{X}_{2}$ et $\mathbf{X}_{3}$. Les incertitudes de mesure de $\varepsilon_{12}$ restent constantes à environ $0,02 \mathrm{~d} \varepsilon$, au cours de la déformation. Par contre, l'incertitude sur le cisaillement $\varepsilon_{23}$, initialement du même ordre de grandeur, augmente progressivement vers $0,1 \mathrm{~d} \varepsilon$ à $\varepsilon_{22}=1$ à cause de la diminution importante de l'épaisseur.

Pour tester la méthode nous citerons les résultats obtenus sur quatre orientations différentes, caractérisées par les indices $(h k l)[u v w]$ où $(h k l)$ 'signifie les indices de Miller du plan de compression de normale $\overline{\mathbf{X}}_{3}$, et [uvw] la direction d'allongement $\mathbf{X}_{2}$.

3.1 Orientations (110) [ $[\overline{1} 1 \overline{2}]$ ET (110) [ $[\overline{1} 1 \overline{1}]$. Pour ces deux orientations les cisaillements $\varepsilon_{23}$ mesurés expérimentalement sont nuls, en accord avec les calculs théoriques, [6] et (A. Skalli, J. Driver et M. Wintenberger, à paraître). Les cisaillements $\varepsilon_{12}$ théoriques et expérimentaux des monocristaux non encastrés sont par contre importants. En effet le cisaillement infinitésimal théorique $\mathrm{d} \varepsilon_{12}$ d'un cristal (110) [1112] non encastré est de $+\sqrt{2} / 4 \mathrm{~d} \varepsilon_{22}\left(\varepsilon_{22}>0\right)$ et la pente initiale de la courbe expérimentale

$$
\tan \alpha\left(=2 \mathrm{~d} \varepsilon_{12}\right)=f\left(\varepsilon_{22}\right)
$$

est proche de la valeur théorique 0,71 (Fig. 4a). De même pour l'orientation (110) $[\overline{1} 1 \overline{1}]$ tan $\alpha \simeq 0,7$ $\varepsilon_{22}$ (Fig. $4 b$ ).

Lorsque ces cristaux sont encastrés par deux autres cristaux, orientés pour supprimer $\varepsilon_{12}$ par une rotation de $\pi$ autour de l'axe $\mathbf{X}_{2}((110)[\overline{1} 1 \overline{2}])$ ou $\mathbf{X}_{1}((110)[\overline{1} 1 \overline{1}])$ le cisaillement $\varepsilon_{12}$ est en effet pratiquement supprimé dans les deux cas (Figs. $4 a$ et $b$ ). La figure 5 montre clairement la différence de mode de déformation entre le monocristal libre et le monocristal encastré d'orientation (110) [1 $1 \overline{1} \overline{2}]$.
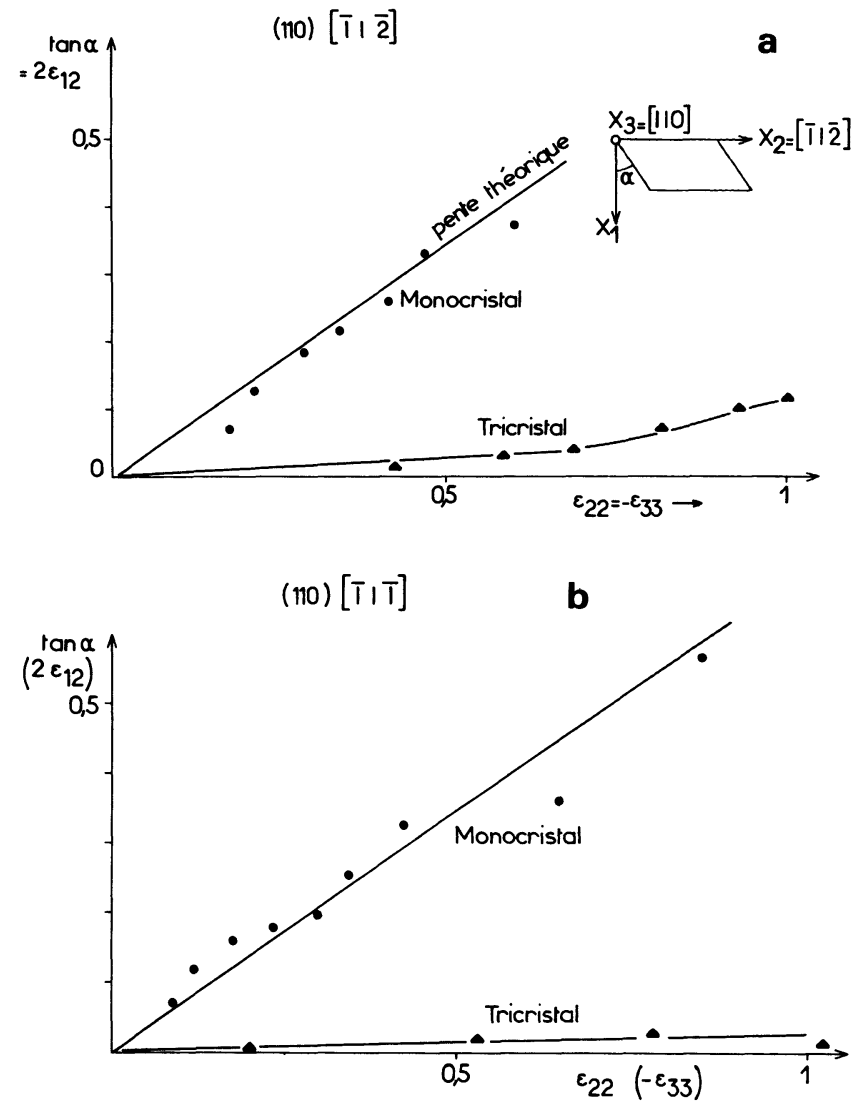

Fig. 4. - Cisaillements $\varepsilon_{12}$ des monocristaux et tricristaux

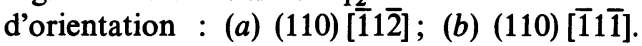

[Shear strains $\varepsilon_{12}$ of the single and tricrystal specimens : (a) $(110)[\overline{1} 1 \overline{2}] ;(b)(110)[\overline{1} 1 \overline{1}]$.

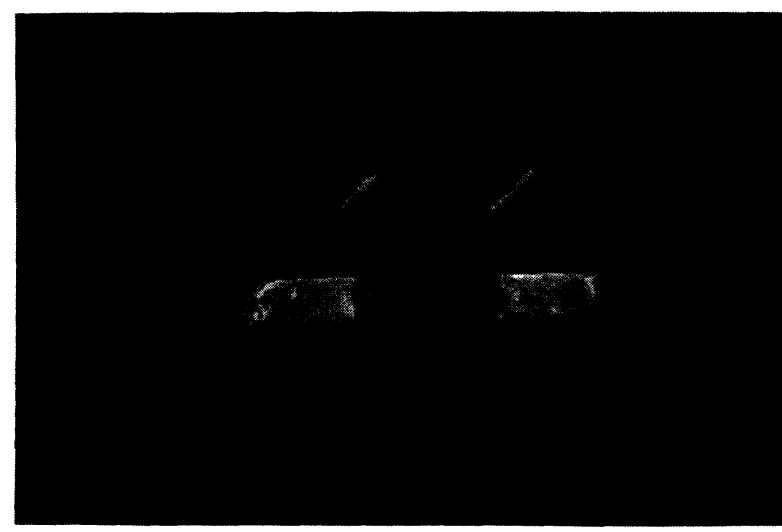

Fig. 5. - Echantillons monocristallin et tricristallin d'orientation $(\overline{1} \overline{1} 0)[\overline{1} 1 \overline{2}]$ après une déformation rationnelle $\varepsilon_{33}$ de -1 .

[Single and tricrystal $(\overline{1} \overline{1} 0)[\overline{1} 1 \overline{2}]$ specimens after a compressive strain $\varepsilon_{33}$ of -1 .]

3.2. ORIENTATIONS $\simeq(159)[90 \overline{1}] \mathrm{ET} \simeq(123)[\overline{1} 11]$. - Ces orientations ne possédant pas de symétrie particulière, la compression plane de monocristaux " libres » conduit à des cisaillements $\varepsilon_{12}$ et $\varepsilon_{23}$ qui sont non nuls. 
Dans le cas de l'orientation $\simeq$ (159) [901] le cisaillement expérimental $\varepsilon_{12}$ est de l'ordre de $-0,13 \varepsilon_{22}$ mais le cisaillement $\varepsilon_{23}$ est relativement important $\left(\varepsilon_{23} \simeq 0,63 \varepsilon_{22}\right)$, figure 6 . Lorsque ce cristal est encastré par deux autres cristaux (tournés de $\pi$ autour de l'axe $\mathbf{X}_{2}$ par rapport au cristal central), le cisaillement $\varepsilon_{23}$ est pratiquement supprimé(Fig. 6) ainsi que le cisaillement $\varepsilon_{12}$.

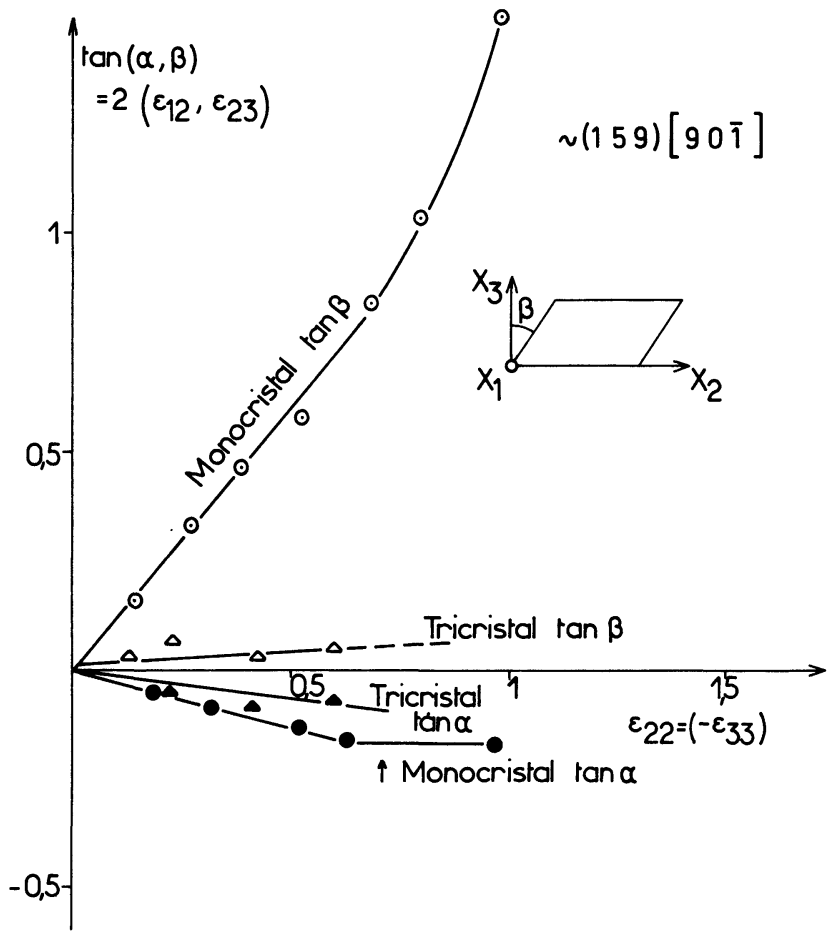

Fig. 6. - Cisaillements $\varepsilon_{12}$ et $\varepsilon_{23}=f\left(\varepsilon_{22}\right)$ de monocristaux et tricristaux d'orientation $\sim(159)$ [901].

[Shear strains $\varepsilon_{12}$ and $\varepsilon_{23}=f\left(\varepsilon_{22}\right)$ of $\sim(159)$ [901] single and tricrystals.]

En ce qui concerne l'orientation (123) [1̄111] les cisaillements mesurés du cristal libre sont respectivement $\varepsilon_{12} \simeq+0,5 \varepsilon_{22}$ et $\varepsilon_{23} \simeq+0,7 \varepsilon_{22}$. Dans ce cas particulier un tricristal a été élaboré tel que la relation d'orientation entre le cristal central et les cristaux d'encastrement soit une rotation de $\pi$ autour de l'axe $\mathbf{X}_{1}$. Rappelons que cette configuration devra permettre de supprimer $\varepsilon_{12}$ mais pas $\varepsilon_{23}$. En effet la figure 7 montre que l'amplitude du cisaillement $\varepsilon_{23}$ du cristal central encastré est initialement égale à celle du monocristal libre. La différence constatée à des déformations importantes, peut être due aux incertitudes des mesures de $\varepsilon_{23}$ dans une éprouvette fortement comprimée. Par ailleurs on notera que le cisaillement $\varepsilon_{12}$ est largement supprimé. Autrement dit, par un choix approprié des relations d'orientation entre cristaux d'encastrement et cristal central nous n'avons imposé que 4 termes du tenseur de déformation plastique $\varepsilon$.

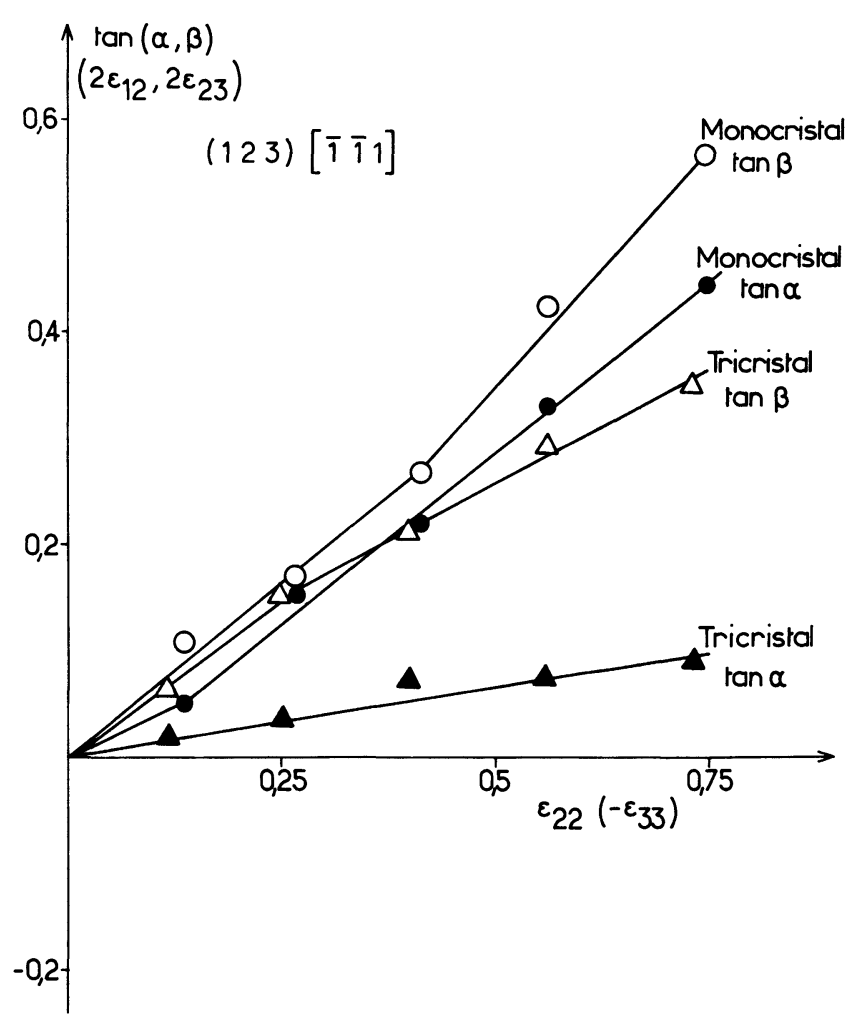

Fig. 7. - Cisaillements $\varepsilon_{12}$ et $\varepsilon_{23}=f\left(\varepsilon_{22}\right)$ de monocristaux et tricristaux d'orientation (123) [1111].

[Shear strains $\varepsilon_{12}$ and $\varepsilon_{23}=f\left(\varepsilon_{22}\right)$ of (123) [11̄1] oriented single and tricrystals.]

4. Remarques et conclusions. - Les résultats expérimentaux confirment l'hypothèse de départ selon laquelle il est possible d'imposer toutes ou une partie du tenseur de déformation plastique d'un cristal en compression plane suivant le mode d'encastrement :

— un monocristal « libre» : 3 composantes indépendantes imposées :

$$
\varepsilon_{11}=\varepsilon_{13}=0, \varepsilon_{33} ;
$$

- un cristal encastré entre deux cristaux orientés par une rotation de $\pi$ autour de $\mathbf{X}_{1}$ (ou $\mathbf{X}_{3}$ ) : 4 composantes indépendantes imposées :

$$
\varepsilon_{11}=\varepsilon_{13}=0, \varepsilon_{33}, \varepsilon_{12}\left(\text { ou } \varepsilon_{23}\right)=0 ;
$$

- un cristal encastré entre deux cristaux orientés par une rotation de $\pi$ autour de $\mathbf{X}_{2}: 5$ composantes indépendantes de $\varepsilon$ imposées.

Par ailleurs, la méthode présente deux avantages importants par rapport aux techniques proposées précédemment (par exemple celle de Hauser et Chalmers [9]); d'une part la mise en cuvre est relativement facile et d'autre part il semble que ce mode d'encastrement soit valable pour des déformations finies allant parfois jusqu'à des valeurs de 1 ou 1,5. 
Grâce à cette technique, des études sont actuellement en cours pour vérifier les théories de plasticité cristalline sous des conditions de déformation complètement ou partiellement imposée.

Remerciements. - Nous remercions bien vivement
MM. C. Goux, Professeur à l'Ecole des Mines de Saint-Etienne et M. Wintenberger, Directeur Scientifique de Péchiney Ugine Kuhlmann, pour l'intérêt qu'ils portent à nos études, ainsi que $\mathrm{P}$. Passet pour son assistance technique. Ces travaux ont été subventionnés par la D.G.R.S.T. et par la Société PUK.

\section{Bibliographie}

[1] Von Mises, R., Z. Angew. Math. Mech. 8 (1928) 161.

[2] TAYLOR, G. I., J. Inst. Met. 62 (1938) 307.

[3] Bishop, J. F. W. et Hill, R., Philos. Mag. 42 (1951) 414 et 1298 .

[4] Renouard, M. et Wintenberger, M., C. R. Hebd. Séan. Acad. Sci. B 292 (1981) 385.

[5] Hosford, W. F., Acta Metall. 14 (1966) 1085.

[6] Chin, G. Y., Nesbitt, E. A. et Williams, A. J., Acta Metall. 14 (1966) 467.
[7] Carpay, F. M. A., Chin, G. Y., Mahajan, S. et Rubin, J. J., Acta Metall. 23 (1975) 1473.

[8] Cyrille, M., Rieux, P. et Passet, P., Second Colloque International "Soudage et Fusion par Faisceau d'électrons ", Avignon (1978), p. 107.

[9] Hauser, J. J. et Chalmers, B., Acta Metall. 9 (1961) 802. 